\title{
Double Right Goronary Artery Arising From Separate Ostia
}

\author{
Emrah Ermis, Emrah Ipek, Selami Demirelli \\ From the Department of Cardiology, Erzurum Training \\ and Research Hospital, Erzurum, Turkey.
}

\section{Abstract:}

We present a rare case of double right coronary artery (RCA) arising from separate ostia. A 62 years old male patient was admitted with acute onset retrosternal chest pain. ECG was suggestive of sinus rhythm with intraventicular conduction delay and pathological q waves in D3 and avF derivations without any significant ST segment elevations. In the coronary angiography, we detected double RCA, each had its own ostium arising separately from right aortic sinus with complete occlusion seen in one RCA. Plaques were present in left coronary arterial system. Guidewires could not be passed in the completely occluded right RCA and conservative therapy was recommended. Double RCA is a congenital coronary anomaly with a potential of atherosclerotic coronary artery disease which needs regular monitoring.

Key words: Coronary Artery Disease, Chest pain, Coronary Angiography, Congenital, Heart Defects, Sinus of Valsalva

\section{Introduction}

Congenital coronary anomalies are seen in $1 \%$ of the general population [1]. Unless diagnosed these anomalies constitute a major problem for invasive cardiologists and cardiovascular surgeons. Double right coronary artery ( $R C A$ ) arising from single or seperate ostia is one of the rarest anomaly in the literature and all of them were mentioned only in case reports [2]. Individuals with double RCA can be unaware of their conditions and are under risk of unpredictable complications due to coronary artery disease or cardiovascular surgery until being diagnosed during coronary angiography or cardiac surgery. Herein we report a patient with double RCA who was admitted to our emergency clinic with the symptoms of unstable angina pectoris. In the coronary angiography we detected double RCA originating from right aortic sinus separately and one of them was totally occluded.

\section{Case Report}

A 62 years old male patient was admitted to our emergency clinic early in the morning with the complaint of retrosternal chest pain of 20 minutes duration. He was a non-smoker and he had a one month history of chest pain, which was sometimes precipitated by effort. His past history was suggestive of hypertension, hyperlipidemia and

\section{Corresponding Author: Dr. Emrah Ermis}

Email: emr_ermis@hotmail.com

Received: April 14, 2014 | Accepted: May 28, 2014 | Published Online: June 15, 2014

This is an Open Access article distributed under the terms of the Creative Commons Attribution License (creativecommons.org/licenses/by/3.0)

Conflict of interest: None declared | Source of funding: Nil | DOl: http://dx.doi.org/10.17659/01.2014.0052 
diabetes mellitus. On physical examination, he was afebrile, had a pulse rate of 88 beats per minute, blood pressure of $140 / 90 \mathrm{mmHg}$ and oxygen saturation of $93 \%$. ECG was in sinus rhythm with intraventicular conduction delay and pathological $q$ waves in D3 and avF derivations without any significant ST segment elevations. Cardiac enzyme levels were within normal limits.

The patient's chest X-ray was normal, while transthoracic echocardiography revealed regional wall motion abnormality in the RCA territory with an ejection fraction of $50 \%$. There was mild mitral regurgitation. He was taken into catheterization laboratory since he had ongoing chest pain with the diagnosis of unstable angina pectoris. His coronary angiography was performed using Judkins technique from the right femoral artery. The RCA was selectively cannulated with a $6 \mathrm{Fr}$ Judkins right diagnostic catheter. In the coronary angiography, we detected double RCA, each had its own ostium arising separately from right aortic sinus and one of them had chronic total occlusion [Fig. 1a-b, 2a]. There were only plaques in the left coronary arterial system [Fig.2b]. The chronic total lesion could not be passed by any guidewires and medical therapy was recommended. After one day coronary intensive care unit monitoring and one day service follow up, he was discharged.

\section{Discussion}

Congenital coronary anomalies are seen in about $1 \%$ of the general population [1]. Double RCA, either originating from single or seperate ostia, is one of the rarest coronary artery anomalies which were mostly mentioned only in some case reports. In the coronary angiography cohort of Yamanaka and collegues [3] with 1,26,595 patients, which is among the most populous coronary angiography series, the incidence of congenital coronary anomalies was $1.6 \%$, however, no double RCA anomaly was reported in this patient cohort. In a

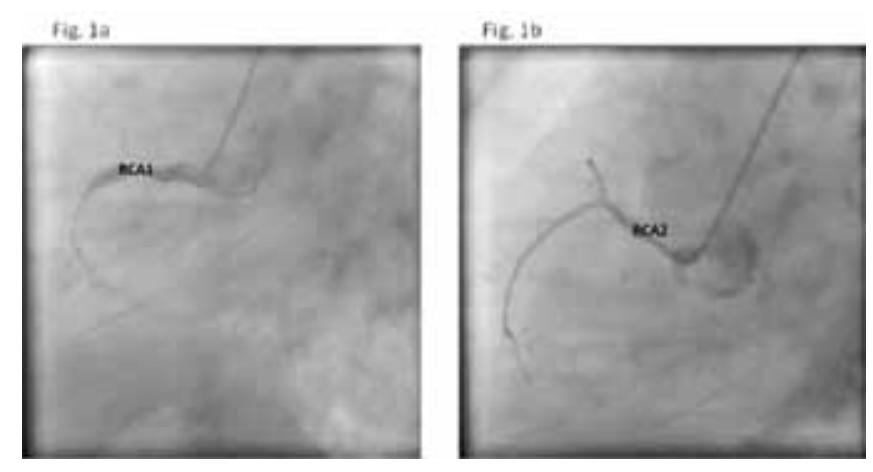

Fig.1 a-b: Right coronary arterial system with double right coronary artery arising from seperate ostia (RCA1 and RCA 2).
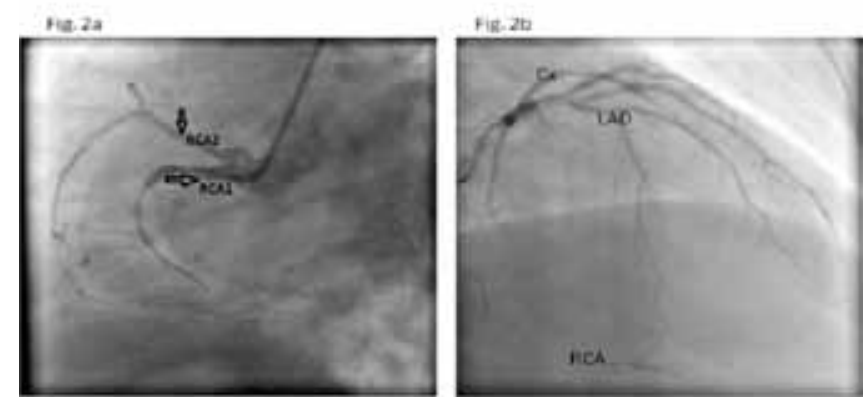

Fig.2a: One of double right coronary arteries had chronic total occlusion, demonstated in percutaneous coronary intervention (RCA 1). Fig.2b: The left coronary arterial system.

wide study of Harikrishnan and collegues [4] with 7400 patients, the incidence of congenital coronary anomaly was $0.46 \%$ and double RCA was detected only in one case (incidence: $0.01 \%$ ). Until now, double RCA was defined as double, dublicated, dual or split and reported 39 times and detected in 46 patients in the literature in which more than the half of the cases were reported from Turkey [2]. Most of these patients had double RCA originated from single ostium, however, 18 of them had double RCA, with separate ostia located in close proximity, in the right coronary sinus. One of the double RCAs has a sinoatrial branch and terminates as posterior descending artery. The other one has a conal and 
right ventricular branches and ends as an acute marginal branch. Double RCA was defined as a benign congenital anomaly in which any specific management was not required. Previously, it was reported that double RCA does not predispose to atherosclerosis or ischemia.

In most of the cases, the first symptom at admission is chest pain. The incidence of stenotic coronary lesions was detected to be more prevalent especially in cases with single than the separate ostia and the prevalance of atherosclerotic coronary artery disease was found to be higher. Previously reported six cases of double RCA presented with acute inferior myocardial infarction [5-10], five of them had single ostium. Although there is not any study documenting any predisposition to acute coronary syndromes until now, it can be postulated that there is an increased risk in cases with single ostium. The cases with double ostia seem to be innocent and increased risk of atherosclerosis in these patients still remains controversial.

Herein, we present a case who was admitted to our emergency clinic with unstable angina pectoris and has a double RCA with double ostia with a chronic total occlusion in one of them detected by the coronary angiography. Other coronary vessels were normal except atherosclerotic plaques. The patient was monitored in coronary intensive care unit for one day and discharged after one day service follow up.

\section{Conclusion}

Double RCA is a congenital coronary anomaly with a potential of atherosclerotic coronary artery disease. Because of this reason, any coronary intervention, aortic root operation and myocardial revascularization in these patients can lead to unexpected complications. Additionally, either treated by invasive procedures or conservative management, all such cases must be monitored.

\section{References}

1. Jacobs ML, Mavroudis C. Anomalies of the coronary arteries: nomenclature and classification. Cardiol Young. 2010;20 Suppl 3:15-19.

2. Chien TM, Chen CW, Chen HM, Lee CS, Lin CC, Chen YF. Double right coronary artery and its clinical implications. Cardiol Young. 2014;24:512.

3. Yamanaka O, Hobbs RE. Coronary artery anomalies in 126,595 patients undergoing coronary arteriography. Cathet Cardiovasc Diagn 1990;21:28-40.

4. Harikrishnan S, Jacob SP, Tharakan J, Titus T, Kumar VK, Bhat A, et al. Congenital coronary anomalies of origin and distribution in adults: a coronary arteriographic study. Indian Heart J 2002;54:271-275.

5. Timurkaynak T, Ciftci $H$, Cengel A. Double right coronary artery with atherosclerosis: a rare coronary artery anomaly. I Invasive Cardiol 2002; 1 4:337-339.

6. Tatli E, Buyuklu M, Altun A, Yilmaztepe M. A patient with double right coronary artery and acute inferior myocardial infarction due to the critical lesion in one of them. Int J Cardiol 2007; 1 19:e30-e31.

7. Rohit M, Bagga S, Talwar KK. Double right coronary artery with acute inferior wall myocardial infarction. J Invasive Cardiol 2008;20:E37-E40.

8. Akcay A, Koroglu S, Kaya H, Koleoglu M, Acar G. An unusual appearance of double right coronary artery. Cardiol Res Pract 2010;201 0:1 23846.

9. Acet $\mathrm{H}$, Ozyurtlu F, Bilik MZ, Ertas F. A rare coronary anomaly:atypical double right coronary artery with an acute inferior myocardial infarction. Korean Circ J 2012;42: 208-21 1.

10. Sawaya FJ, Sawaya Jl, Angelini P. Split right coronary artery: its definition and its territory. Tex Heart Inst J 2008;35:477-479. 